\title{
Anti-Müllerian Hormone and Ovarian Morphology in Women With Hypothalamic Hypogonadism
}

\author{
Amoon Alemyar, Anne-Lotte L.F. van der Kooi, and Joop S.E. Laven \\ Division of Reproductive Endocrinology and Infertility, Department of Obstetrics and Gynecology, Erasmus \\ University Medical Centre, 3015 GD Rotterdam, The Netherlands
}

ORCiD numbers: 0000-0003-3056-1581 (A. Alemyar); 0000-0003-0497-3814 (A.-L. L.F. van der Kooi); 0000-0002-5444-9845 (J. S.E. Laven).

Context: Different phenotypical features of women with hypothalamic hypogonadism (HH), also known as World Health Organization-1 anovulation, including ovarian morphology, have been scarcely described in large cohorts. Some studies have reported increased levels of antiMüllerian hormone (AMH) in women with $\mathrm{HH}$.

Objective: To assess whether women with $\mathrm{HH}$, compared with healthy controls, have increased serum levels of $\mathrm{AMH}$ and what proportion of these women erroneously meet the Rotterdam Criteria for Polycystic Ovarian Syndrome (PCOS).

Design, Setting and Participants: Retrospective cohort study in a Dutch academic medical center including 83 women with neither anovulation nor menstrual cycle disorders (healthy controls), 159 women with $\mathrm{HH}$ and 3640 women with PCOS. Age matching was used between the $\mathrm{HH}$ and PCOS group (1:2 ratio) to create a second group consisting of 318 age-matched women with PCOS.

Intervention: None.

Main outcome measures: AMH levels and ovarian morphology.

Results: Median AMH serum levels for the HH group were $3.8(<0.1-19.8)$, compared with 7.5 $(<0.1-81.0)$ in the PCOS group and $1.9(<0.1-21.5)$ in the control group $(P<0.001)$. In the $\mathrm{HH}$ group, 58 (36\%) erroneously met the Rotterdam Criteria for PCOS (meeting 2 of 3 criteria).

Conclusions: $\mathrm{AMH}$ levels are increased in women with $\mathrm{HH}$. We hypothesize that this increase, although there was no increase in follicle count, may be explained by the presence of a relatively large pool of antral follicles smaller than $2 \mathrm{~mm}$ in diameter, that are undetectable by transvaginal ultrasound. This study highlights the importance of measuring gonadotropins and estradiol before diagnosing a patient with PCOS. (J Clin Endocrinol Metab 105: e2008-e2015, 2020)

Keywords: anti-Müllerian hormone, hypothalamic hypogonadism, PCOS, ovarian function, ovarian morphology

A novulation is the state in which a woman does not ovulate, often resulting in oligomenorrhea or amenorrhea (1). The World Health Organization

ISSN Print 0021-972X ISSN Online 1945-7197

Printed in USA

(C) Endocrine Society 2020. All rights reserved. For permissions, please e-mail: journals. permissions@oup.com

Received 3 December 2019. Accepted 12 March 2020.

First Published Online 14 March 2020

Corrected and Typeset 31 March 2020.
(WHO) identifies 3 distinct groups: WHO-1 is characterized by dysfunction of the hypothalamic-pituitary axis to produce gonadotropins ( $\mathrm{LH}$ and $\mathrm{FSH})$ resulting

Abbreviations: $\mathrm{AMH}$, anti-Müllerian hormone; BMI, body mass index; E2, estradiol; FAI free androgen index; FNPO, follicle number per ovary; $\mathrm{HH}$, hypothalamic hypogonadism; PCOM, polycystic ovarian morphology; PCOS, polycystic ovary syndrome; WHO, World Health Organization 
in anovulation and in low estrogens. This category of anovulation is also known as hypothalamic hypogonadism $(\mathrm{HH})(2,3)$. HH may be caused by excessive exercise or physical stress, or from eating disorders such as anorexia nervosa or bulimia (4). In about $5 \%$ to $10 \%$ of all anovulatory women, $\mathrm{HH}$ seems to be the cause (3). Patients with $\mathrm{HH}$ are at risk of osteoporosis $(5,6)$ and cardiovascular disease $(7,8)$, as a result of their persistent low estrogen levels (7).

By far the largest part of women with anovulation are classified in the WHO-2 category, which is characterized by normal FSH and estradiol $\left(\mathrm{E}_{2}\right)$ levels. Most women in this category can be classified as having polycystic ovary syndrome (PCOS). In these women, irregular cycles are accompanied by hyperandrogenism and polycystic ovarian morphology (PCOM) (9-11).

Anti-Müllerian hormone (AMH) is produced in the ovary by granulosa cells of small growing pre-antral and antral follicles. AMH inhibits the recruitment of primordial follicles out of the primordial follicle pool, decreases FSH sensitivity of individual follicles, and inhibits aromatase activity (consequently increasing androgen levels in the ovary) $(12,13)$. Because the ovarian follicle pool gradually diminishes over the course of a women's reproductive lifetime, a simultaneous decrease in AMH levels occurs $(14,15)$.

In the past, higher follicle number per ovary (FNPO) in women with $\mathrm{HH}$ compared with controls has been reported (16). This may lead to the suspicion that, when follicle count reaches the number required for the characterization of PCOM, women with $\mathrm{HH}$ may be misdiagnosed with PCOS, if gonadotropin levels FSH and estrogen serum levels are not taken into account (17).

The primary goal of this paper is to assess whether women with $\mathrm{HH}$ have increased AMH serum levels compared with women with regular cycles and compared with women with PCOS. The secondary goal of this paper is to assess whether PCOM occurs more often in women with $\mathrm{HH}$ than in women with regular cycles and in women with PCOS. Finally, we wanted to evaluate how often the negligence of measuring serum levels of FSH, $\mathrm{LH}$, and $\mathrm{E}_{2}$ would falsely lead to a PCOS diagnosis instead of the correct $\mathrm{HH}$ diagnosis.

\section{Materials and Methods}

\section{Study design}

In this retrospective single-center study, patients with cycle disorders and anovulation who visited the reproductive endocrinology and infertility clinic of the Erasmus University Medical Center in Rotterdam, The Netherlands, were consecutively selected between 1993 and 2018. Participants were not on fertility medication during this visit; in case of oral contraceptive usage, they were seen at day 7 in their pill-free week. During their visit, extensive patient history, general health, family history, previous and current use of medication, and self-reported ethnicity was recorded. During physical examination, anthropometric measurements (e.g., weight, height, body-mass index [BMI]) were assessed. Transvaginal ultrasonography was performed to assess the number of antral follicles sized 2 to $10 \mathrm{~mm}$ in diameter, as well as to assess the ovarian volume in the left and right ovary. Laboratory blood tests were drawn randomly in the anovulatory cycles (fasting blood samples were taken with venipuncture) to collect data on endocrine and metabolic profiles. Blood samples were processed within 2 hours. Serum was produced and isolated by centrifuging blood samples at $3000 \mathrm{rpm}$ for a duration of 10 minutes at $20^{\circ} \mathrm{C}$.

\section{Patient selection}

$\mathrm{HH}$ was defined as having low levels of gonadotropins (FSH and $\mathrm{LH}<2.0 \mathrm{U} / \mathrm{L})$, low levels of $\mathrm{E}_{2}(<100 \mu \mathrm{mol} / \mathrm{L})$, and either oligomenorrhea (menstrual periods being between 35 days and 6 months apart) or amenorrhea (no menstruation for at least 6 months).

PCOS was defined as having normal levels of FSH, LH, and estrogen in addition to meeting 2 of the 3 criteria in the Rotterdam Criteria (1) oligomenorrhea/amenorrhea, (2) PCOM (defined as $\geq 12$ follicles measuring 2 to $9 \mathrm{~mm}$ in diameter in at least 1 ovary), and/or (3) signs of hyperandrogenism (17). In accordance to the Rotterdam Criteria for PCOS, hyperandrogenism was assessed and classified as either biochemical or clinical hyperandrogenism (hirsutism). Biochemical hyperandrogenism was defined by the free androgen index (FAI) on the calculation of (testosterone/SHBG) $\times 100(18)$. Biochemical hyperandrogenism was defined as having a FAI higher than 2.9. Clinical hyperandrogenism was assessed visually using the modified Ferriman-Gallwey score $(19,20)$, a standardized visual scale. Clinical hyperandrogenism was defined as a modified Ferriman-Gallwey score of 5 or higher (18).

Healthy control women were selected from ongoing cohort studies at our department where, in addition to having a regular cycle pattern and no oligomenorrhea or amenorrhea, having normal levels of LH, FSH and estrogen as well as testosterone were required (21). Laboratory blood tests for the control group were drawn at days 3 through 5 of their natural cycle or seventh day of their pill-free week. This study was approved by the Medical Ethics Review Committee (institutional review board protocol number 2016-216, EMC) and written informed consent was obtained from all participants.

\section{Hormone assays}

Patient samples were stored at $-20^{\circ} \mathrm{C}$ before hormone levels were determined. AMH levels were determined using double-antibody ELISA (Beckman Coulter, Inc., AMH Gen II assay, Webster, TX) (3). In patients who had their AMH levels measured before 2011, determination of AMH levels was done with an ultrasensitive ELISA (Immunotech-Coulter, Marseilles, France) $(15,22)$. These measurements were batch by batch-adjusted for comparability to AMH levels measured with the newer ELISA technology.

FSH, LH, and SHBG were determined using the Immulite platform assay. $\mathrm{E}_{2}$ was determined using radioimmunoassay (DPC). Androstenedione was determined using the Immulite 2000 assay and the liquid chromatography tandem mass 
spectometry assay. Testosterone was determined using liquid chromatography tandem mass spectometry.

Intra-assay and inter-assay coefficient variations for the Beckman Coulter Inc. Gen II assay AMH assay were $<5 \%$ and $<10 \%$ (3), for the Immunotech-Coulter AMH assay, these were $<5 \%$ and $8 \%$, respectively $(15,22)$. Intra-assay and inter-assay coefficient variations for $\mathrm{FSH}$ were $<3.0 \%$ and $<6.2 \%$, for $\mathrm{LH}<3.5 \%$ and $<6.4 \%$, for $\mathrm{E}_{2}<11 \%$ and $<11 \%$, for testosterone $<3 \%$ and $<5 \%$, for androstenedione $<8 \%$ and $<11 \%$, and for SHBG, respectively, $<4 \%$ and $<5 \%(23)$.

\section{Statistical analysis}

IBM SPSS Statistics 25 was used for statistical analysis. Continuous variables were presented as mean and standard deviation. Independent $t$-tests were used when comparing continuous variables that were normally distributed. Continuous variables that were not normally distributed were assessed using the Mann-Whitney $U$ test, where these variables were presented as median and range. Normality was assessed using the Kolmogorov-Smirnov test. Categorical variables were presented as numbers and percentages. Differences between categorical variables such as elevated hormone levels were assessed using a $\chi^{2}$ test. If a cell contained less than 5 individuals, Fischer's exact test was used for assessing the difference in categorical variables. Rstudio version 1.1.463 in combination with the packages "Matchit" (https://cran.r-project.org/web/ packages/MatchIt/index.html) and "foreign" (https://cran.rproject.org/web/packages/foreign/index.html) were used to age match every $\mathrm{HH}$ patient with 2 PCOS patients (1:2 ratio). $P$ values $<0.05$ were considered statistically significant.

\section{Results}

\section{Study population}

We identified 159 women with $\mathrm{HH}$ and 3640 women with PCOS. The control group consisted of 83 women with no cycle disorders or anovulation. We age-matched 318 PCOS patients to the 159 patients in the $\mathrm{HH}$ group; this group will be referred to as the age-matched PCOS group.

The $\mathrm{HH}$ group did not statistically differ in age from the control group $(26.67 \pm 6.67$ vs $28.37 \pm 5.86)$. The PCOS group $(\mathrm{N}=3640)$ was significantly older $(27.62 \pm 5.43)$ compared with the $\mathrm{HH}$ group $(P<0.05)$, but did not statistically differ in age from the control group.

The HH group's BMI $(23.06 \pm 5.78)$ did not statistically differ from that of the control group $(23.55 \pm 3.74)$. The PCOS group had a higher BMI $(26.95 \pm 6.50)$ compared with both the HH $(P<0.001)$ and control group $(P<0.01)$. The increased BMI levels in the PCOS group persisted after age matching with the $\mathrm{HH}$ group $(\mathrm{N}=318$, BMI $27.07 \pm 6.46)$ (Table 1).

\section{AMH levels and ovarian morphology}

Median AMH serum levels were significantly higher in the $\mathrm{HH}$ group compared with the control group (medians 3.8 vs $1.9 \mu \mathrm{g} / \mathrm{L}, P<0.001)$, but lower than $\mathrm{AMH}$ levels in the PCOS group (median AMH $7.5 \mu \mathrm{g} / \mathrm{L}$ ) (Fig. 1A and Table 2). After age-matching, the difference in $\mathrm{AMH}$ levels between the PCOS group and $\mathrm{HH}$ group decreased. In the age-matched PCOS group, median AMH levels were $6.7 \mu \mathrm{g} / \mathrm{L}$. These AMH levels remained significantly higher compared with both the $\mathrm{HH}$ group and the control group $(P<0.001)$ (Table 2$)$.

Antral follicle count was not statistically different in the HH group compared with the control group (20.5 [range 4.0-109.0] vs 21.5 [range 4.0-70.0]) (Table 2

\section{Table 1. Patient's characteristics}

\begin{tabular}{lcccc}
\hline & Controls & HH & Age-Matched PCOS & PCOS \\
\hline $\mathrm{N}$ & 83 & 159 & 318 & 3640 \\
Age (years) & $28.37 \pm 5.86$ & $26.67 \pm 6.67$ & $26.66 \pm 6.63^{a}$ & $27.62 \pm 5.43^{b}$ \\
BMI (kg/m $\left.{ }^{2}\right)$ & $23.55 \pm 3.74$ & $23.06 \pm 5.78$ & $27.07 \pm 6.46^{c, d}$ & $26.95 \pm 6.50^{c, d}$ \\
Height (cm) & $170.67 \pm 7.32$ & $168.32 \pm 8.06$ & $167.02 \pm 7.62^{c}$ & $167.13 \pm 7.56^{c}$ \\
Weight (kg) & $69.00 \pm 14.27$ & $65.38 \pm 17.73$ & $75.55 \pm 19.06^{a, d}$ & $75.25 \pm 18.76^{a, e}$ \\
Insulin (pmol/L) & $54.25 \pm 27.32$ & $60.67 \pm 67.52$ & $84.14 \pm 76.65^{e, f}$ & $84.54 \pm 147.39^{b}$ \\
FSH (IU/L) & $6.33 \pm 2.49$ & $3.09 \pm 2.23^{e}$ & $5.25 \pm 2.23^{d, f}$ & $5.56 \pm 2.52^{c, e}$ \\
LH (IU/L & $3.78 \pm 1.60$ & $0.84 \pm 0.90^{e}$ & $8.86 \pm 7.07^{d, f}$ & $9.58 \pm 8.96^{d, f}$ \\
E $_{2}$ (pmol/L) & $169.60 \pm 108.86$ & $72.31 \pm 119.09^{e}$ & $270.08 \pm 198.79^{d, f}$ & $278.59 \pm 225.07^{d, f}$ \\
Testosterone (nmol/L) & $1.15 \pm 0.51$ & $0.78 \pm 0.42^{e}$ & $1.91 \pm 0.93^{d, f}$ & $1.95 \pm 1.04^{d, f}$ \\
Androstenedione & $3.48 \pm 1.20$ & $3.05 \pm 1.46$ & $6.16 \pm 2.59^{d, f}$ & $6.14 \pm 2.70^{d, f}$ \\
SHBG (nmol/L) & $71.43 \pm 44.13$ & $62.88 \pm 48.75$ & $45.37 \pm 29.77^{d, f}$ & $46.67 \pm 29.85^{d, f}$ \\
Free androgen index & $1.47 \pm 0.78$ & $1.67 \pm 1.79$ & $5.50 \pm 4.38^{d, f}$ & $5.65 \pm 5.30^{d, f}$ \\
Ferriman-Gallwey score & $3.45 \pm 1.97$ & $4.64 \pm 3.85$ & $6.92 \pm 5.14^{a, e}$ & $7.29 \pm 5.55^{a, e}$ \\
\hline
\end{tabular}

Values represent the mean \pm standard deviation.

$\mathrm{BMI}$, body mass index, $\mathrm{E}_{2}$, estradiol; PCOS, polycystic ovarian syndrome.

${ }^{a} P<0.05$ compared with controls.

${ }^{b} P<0.05$ compared with $\mathrm{HH}$.

${ }^{c} P<0.01$ compared with controls

${ }^{d} P<0.001$ compared with $\mathrm{HH} P$ values obtained through independent-samples $t$-test.

${ }^{e} P<0.01$ compared with $\mathrm{HH}$.

${ }^{f} P<0.001$ compared with controls. 
and Fig. 1B). When looking at the follicle counts in each ovary separately, no differences were found in follicle counts. Similarly, no statistical difference was found for ovarian volume. However, the PCOS group showed increased antral follicle count (36.0, range 4.0-220.0) and total ovarian volume (17.52, range 1.26-200.62) compared with both the HH group and control group $(P<0.001)$. In the age-matched PCOS group, significantly higher antral follicle count and total ovarian volume were also found compared with both the $\mathrm{HH}$ and control group, but were not higher than found in the PCOS group.

Table 3 shows that PCOM did not occur more often in the $\mathrm{HH}$ group compared with the control group ( $31.4 \%$ vs $24.1 \%$ ). In the PCOS group, PCOM $(87.5 \%)$ occurred more often compared with both the $\mathrm{HH}$ group and control group $(P<0.001)$. After age-matching, the difference between these groups remained large; PCOM

A

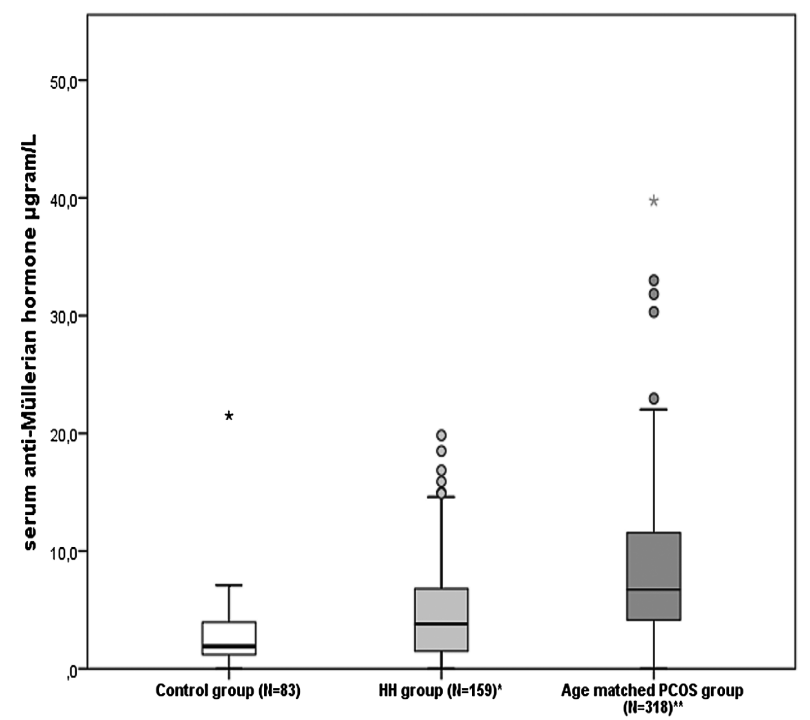

occurred in $81.4 \%$ of the patients in the age-matched PCOS group.

\section{Hyperandrogenism}

Testosterone levels in the PCOS group and agematched PCOS group $(1.95 \pm 1.04 \mathrm{nmol} / \mathrm{L}$ and $1.91 \pm 0.93 \mathrm{nmol} / \mathrm{L}$, respectively) were higher compared with both the $\mathrm{HH}$ group and control group $(0.78 \pm 0.42$ and $1.15 \pm 0.51 \mathrm{nmol} / \mathrm{L}$, respectively, $P<0.001)$.

SHBG and androstenedione levels did not statistically differ in the $\mathrm{HH}$ group compared with the control group. In the PCOS and age-matched PCOS group, SHBG was significantly lower, whereas androstenedione levels were significantly higher compared with both the HH group and the control group (Table 1).

Biochemical hyperandrogenism did not occur more often in the $\mathrm{HH}$ group compared with the

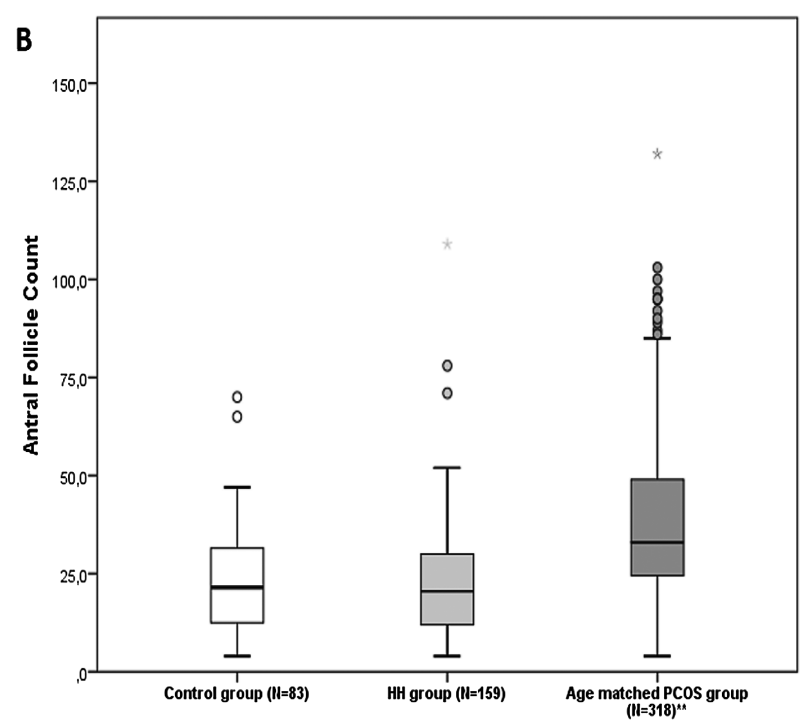

Figure 1. (A) Boxplot showing serum anti-Müllerian hormone levels in the control, HH, and age-matched PCOS group. (B) Boxplot showing antral follicle count in the control, $\mathrm{HH}$, and age-matched PCOS group. ${ }^{*} P<0.001$ compared with the control group. ${ }^{*} P<0.001$ compared with the control group and the $\mathrm{HH}$ group.

\section{Table 2. Patient's characteristics}

\begin{tabular}{|c|c|c|c|c|}
\hline & Controls & $\mathrm{HH}$ & Age-Matched PCOS & PCOS \\
\hline $\mathbf{N}$ & 83 & 159 & 318 & 3640 \\
\hline$\overline{\mathrm{AMH}(\mu \mathrm{g} / \mathrm{L})^{a}}$ & $1.9(<0.1-21.5)$ & $3.8(<0.1-19.8)^{b}$ & $6.7(<0.1-39.8)^{b, c}$ & $7.5(<0.1-81.0)^{b, c}$ \\
\hline Follicle count right ovary & $10.0(2.0-32.0)$ & $9.0(2.0-57.0)$ & $17.0(2.0-67.0)^{b, c}$ & $18.0(2.0-110.0)^{b, c}$ \\
\hline Follicle count left ovary & $9.5(2.0-43.0)$ & $9.0(2.0-52.0)$ & $16.0(2.0-65.0)^{b, c}$ & $17.00(2.0-115.0)^{b, c}$ \\
\hline Antral follicle count & $21.5(4.0-70.0)$ & $20.5(4.0-109.0)$ & $33.0(4.0-132.0)^{b, c}$ & $36.0(4.0-220.0)^{b, c}$ \\
\hline Volume right ovary & $4.95(1.26-23.57)$ & $3.71(0.10-40.67)$ & $8.90(0.80-50.59)^{b, c}$ & $9.16(0.53-131.33)^{b, c}$ \\
\hline Total ovarian volume & $9.98(2.03-50.25)$ & $8.46(0.78-53.45)$ & $16.71(2.89-130.74)^{b, c}$ & $17.52(1.26-200.62)^{b, c}$ \\
\hline
\end{tabular}

Values represent the median (range).

$\mathrm{AMH}$, anti-Müllerian hormone; $\mathrm{HH}$, hypothalamic hypogonadism; PCOS, polycystic ovary syndrome.

${ }^{a}$ The lower limit of detection for AMH was $0.1 \mu \mathrm{g} / \mathrm{L}$.

${ }^{b} P<0.001$ compared with controls.

${ }^{c} P<0.001$ compared with HH $P$ values obtained through Mann-Whitney $U$ test. 
Table 3. Biochemical and clinical hyperandrogenism given as numbers and percentages among the study groups

\begin{tabular}{lcccr}
\hline & Controls & HH & Age-Matched PCOS $^{a}$ & PCOS $^{\text {N }}$ \\
$\mathbf{N}$ & 83 & 159 & 318 & 3640 \\
\hline Biochemical hyperandrogenism & $3(3.6 \%)$ & $8(5.0 \%)$ & $182(57.2 \%)^{c, d}$ & $1972(54.2 \%)^{c, d}$ \\
Clinical hyperandrogenism score & $2(2.4 \%)$ & $16(10.1 \%)^{b}$ & $98(30.8 \%)^{c, d}$ & $986(27.1 \%)^{c, d}$ \\
Polycystic ovarian morphology & $20(24.1 \%)$ & $50(31.4 \%)$ & $259(81.4 \%)^{d, e}$ & $3185(87.5 \%)^{d, e}$ \\
\hline
\end{tabular}

Values represent the number (percentage) of women. Biochemical hyperandrogenism was defined as Free-androgen-index > 2.9; clinical hyperandrogenism was defined as Ferriman-Gallwey score $\geq 5$; pPolycystic ovarian morphology was defined as having 12 or more antral follicles in the left or right ovary.

${ }^{a}$ Age matched with $\mathrm{HH}$ group.

${ }^{b} P<0.05$ compared to controls (Fischer's Exact Test)

${ }^{c} P<0.001$ compared to controls (Fischer's Exact Test).

${ }^{d} P<0.001$ compared to HH (Pearson's $X^{2}$ test).

${ }^{e} P<0.001$ compared to controls (Pearson's $X^{2}$ test).

control group. In the HH group, 5.0\% had biochemical hyperandrogenism compared with $3.6 \%$ in the control group. Clinical hyperandrogenism did occur more often in the $\mathrm{HH}$ group compared to the control group: in the $\mathrm{HH}$ group, $10.1 \%$ had clinical hyperandrogenism compared with $2.4 \%$ in the control group $(P<0.05)$ (Table 3).

In our control group, we found that $3.6 \%$ (3 patients) had biochemical hyperandrogenism but the entire group had normal testosterone levels: this was caused by low SHBG levels in specifically these 3 patients resulting in a high FAI.

\section{Rotterdam Criteria for PCOS in women with HH}

In the $\mathrm{HH}$ group, $58(36 \%)$ women met the Rotterdam Criteria for PCOS, if gonadotropin and estrogen levels were not taken into account. The phenotypes for meeting the Rotterdam Criteria for PCOS in the $\mathrm{HH}$ group were: $39(24.5 \%)$ had PCOM and amenorrhea/oligomenorrhea, $11(6.9 \%)$ had amenorrhea/oligomenorrhea and hyperandrogenism, and 8 $(5.0 \%)$ had PCOM, amenorrhea/oligomenorrhea and hyperandrogenism.

As for the control group: only 1 patient met the Rotterdam Criteria for PCOS. This patient had both PCOM and hyperandrogenism.

\section{Discussion}

This large study shows that AMH serum levels are increased in women with $\mathrm{HH}$ compared with healthy regularly cycling ovulatory controls, but that their levels are lower than those of women with PCOS. Antral follicle count and total ovarian volume are, however, not increased in women with $\mathrm{HH}$ compared with healthy controls.
These findings are in discordance with results from a small study including 28 women with $\mathrm{HH}$ (16), which reported higher AMH levels and higher FNPO in women with $\mathrm{HH}$ compared with controls. However, of these 28 women, only 11 had validated data available for FNPO. This same group (24) also reported that women with $\mathrm{HH}$ had higher AMH levels and a higher mean ovarian volume compared with controls. These findings are also in discordance with results from a study published by Luisi et al (25)., in which no significant difference in AMH was found between a group of women with hypothalamic amenorrhea and a group of women consisting of healthy controls.

We hypothesize that the increase in $\mathrm{AMH}$ levels in the $\mathrm{HH}$ group compared with the control group in the current study, whereas there was no increase in follicle count, can be explained by the presence of a relatively large pool of antral follicles $<2 \mathrm{~mm}$ in diameter. These follicles are not counted during transvaginal ultrasonography although they do secrete AMH. Support for this explanation is found in the study performed by Weenen et al., (26) in which, after staining with AMH antibodies, histological specimens showed considerable AMH expression in antral follicles $<2 \mathrm{~mm}$ in diameter. Moreover, although we did not find an increase in antral follicles in the $\mathrm{HH}$ group, increased numbers of antral follicles may not necessarily be required for elevated AMH levels. Pellatt et al. (27) suggest that AMH production per antral follicle may be increased in different pathological conditions leading to anovulation and could drive increased levels of AMH. This may also explain the higher AMH levels with similar follicle counts, as was also found in the study by Jonard et al. (28). On the 1 hand, the authors of this study suggest that increased $\mathrm{AMH}$ production per antral follicle might indeed be the cause for increased AMH levels 
without a coincident increase in antral follicle count in women with hypothalamic amenorrhea. On the other hand, they also took into consideration the possibility of an increased number of small follicles undetectable on ultrasound because of their size.

Another explanation might be that the follicles that produce large amounts of AMH are the healthiest ones and therefore they will respond better to exogenous gonadotropins. Indeed, the predictive value of $\mathrm{AMH}$ levels in women with $\mathrm{HH}$ has been reported by van Elburg et al. They showed that as baseline levels of AMH increased, so did the probability of weight increase and recovery of menstrual cycles (29).

In line with the similar number of follicles in women from the HH group and control group, PCOM did not occur more often in women with $\mathrm{HH}$ compared with healthy ovulatory controls. Indeed, similar findings were reported in a study performed by Lie Fong et al. (3).

Biochemical hyperandrogenism did not occur more often in women with $\mathrm{HH}$ than in the control group, and testosterone levels were in fact, as to be expected (30), lower in women with HH. However, clinical hyperandrogenism did occur more often in women with $\mathrm{HH}$ than in healthy controls. Indeed, women with $\mathrm{HH}$ may experience, for example, acne, alopecia, and/ or hirsutism (18), and physicians should be aware of these symptoms and discuss this with their patient. Moreover, treatment of these distressing complaints can be important steps in optimizing quality of life in these women. That clinical hyperandrogenism was observed more often in the HH group without an obvious increase in FAI may indicate disturbances at the level of the androgen receptor or androgen processing. Future research is warranted to explore this finding.

Interestingly, we found that more than one-third of the $\mathrm{HH}$ group met the Rotterdam Criteria for PCOS. Indeed, other studies (31) support this finding, showing that $86 \%$ women with $\mathrm{HH}$ met the Rotterdam Criteria for PCOS. This stresses the need for a correct distinction between $\mathrm{HH}$ and PCOS because false-positive diagnoses may lead to employment of treatment options that are suboptimal for these women.

In the latest guidelines for diagnosing PCOS (18), a cutoff criterion for PCOM is described as 20 antral follicles per ovary, instead of the 12 antral follicles per ovary we have used as a cutoff. Because our employed ultrasound had a capacity of 4 to $8 \mathrm{MHz}$, (and the guideline changed the threshold of PCOM because of improving technologies with improving bandwidth $>8 \mathrm{MHz}$ ), we chose to maintain the analyses using the cutoff of 12 antral follicles per ovary. Regardless, when changing the cutoff to 20 antral follicles per ovary, we found that $20.3 \%$ met the Rotterdam Criteria for PCOS. That is still more than one-fifth of women who have hypothalamic hypogonadism.

In light of recent debate whether the cumbersome ultrasound assessment of PCOM might be replaced in time by a single AMH measurement (12), the need for initial assessment of $\mathrm{FSH}, \mathrm{LH}$, and $\mathrm{E}_{2}$ levels becomes even more important. In particular, those women with $\mathrm{HH}$ and elevated levels of AMH will be erroneously misdiagnosed as having PCOS.

In conclusion, we report increased AMH levels in women with $\mathrm{HH}$ compared with healthy controls, and we therefore stress the importance of measuring gonadotropins and $\mathrm{E}_{2}$ in the diagnostic workup of anovulatory patients because AMH and sonographic data are not specific enough to distinguish between PCOS and $\mathrm{HH}$.

\section{Acknowledgments}

The authors thank Olivier van Valkenburg, Sharon Lie Fong, Wendy van Dorp, Yvonne Louwers, Pauline Wijngaard, Cindy Meun, Rivka Koedooder, Eva van Marion, and Alexandra Dietz-de Loos for their contribution in the screening of all included patients and Hester Pastoor and Lizka Nekrui for data management.

\section{Additional Information}

Correspondence and Reprint Requests: Anne-Lotte van der Kooi, Division of Reproductive Endocrinology and Infertility, Department of Obstetrics and Gynecology, Erasmus University Medical Centre, 3015 GD Rotterdam, The Netherlands. E-mail: a.vanderkooi@erasmusmc.nl

Disclosure Summary: J.S.E.L. has received fees or grant support in the most recent 5-year period from the following organizations (in alphabetical order): Dutch Heart Foundation, Dutch Medical Research Counsel (ZonMW), Euroscreen/ Ogeda, Ferring, Netherland Genomic Initiative (NGI), Roche Diagnostics, Danone, and Titus Healthcare.

Data Availability: Restrictions apply to the availability of data generated or analyzed during this study to preserve patient confidentiality or because they were used under license. The corresponding author will on request detail the restrictions and any conditions under which access to some data may be provided.

\section{References and Notes}

1. Hamilton-Fairley D, Taylor A. Anovulation. BMJ. 2003;327(7414):546-549.

2. Anovulatory infertility. The ESHRE Capri Workshop Group. Hum Reprod. 1995;10(6):1549-1553.

3. Lie Fong S, Schipper I, Valkenburg O, de Jong FH, Visser JA, Laven JS. The role of anti-Müllerian hormone in the classification 
of anovulatory infertility. Eur J Obstet Gynecol Reprod Biol. 2015;186:75-79.

4. Meczekalski B, Podfigurna-Stopa A, Warenik-Szymankiewicz A, Genazzani AR. Functional hypothalamic amenorrhea: current view on neuroendocrine aberrations. Gynecol Endocrinol. 2008;24(1):4-11.

5. Grinspoon S, Miller K, Coyle C, et al. Severity of osteopenia in estrogen-deficient women with anorexia nervosa and hypothalamic amenorrhea. J Clin Endocrinol Metab. 1999;84(6):2049-2055.

6. Vescovi JD, Jamal SA, De Souza MJ. Strategies to reverse bone loss in women with functional hypothalamic amenorrhea: a systematic review of the literature. Osteoporos Int. 2008;19(4):465-478.

7. Meczekalski B, Katulski K, Czyzyk A, Podfigurna-Stopa A, Maciejewska-Jeske M. Functional hypothalamic amenorrhea and its influence on women's health. J Endocrinol Invest. 2014;37(11):1049-1056.

8. Bairey Merz CN, Johnson BD, Sharaf BL, et al.; WISE Study Group. Hypoestrogenemia of hypothalamic origin and coronary artery disease in premenopausal women: a report from the NHLBIsponsored WISE study. J Am Coll Cardiol. 2003;41(3):413-419.

9. Pigny P, Merlen E, Robert Y, et al. Elevated serum level of antimullerian hormone in patients with polycystic ovary syndrome: relationship to the ovarian follicle excess and to the follicular arrest. J Clin Endocrinol Metab. 2003;88(12):5957-5962.

10. Franks S, Stark J, Hardy K. Follicle dynamics and anovulation in polycystic ovary syndrome. Hum Reprod Update. 2008;14(4):367-378.

11. Laven JS, Mulders AG, Visser JA, Themmen AP, De Jong FH, Fauser BC. Anti-Müllerian hormone serum concentrations in normoovulatory and anovulatory women of reproductive age. $J$ Clin Endocrinol Metab. 2004;89(1):318-323.

12. Teede H, Misso M, Tassone EC, et al. Anti-müllerian hormone in PCOS: a review informing international guidelines. Trends Endocrinol Metab. 2019;30(7):467-478.

13. Pellatt L, Rice S, Dilaver N, et al. Anti-Müllerian hormone reduces follicle sensitivity to follicle-stimulating hormone in human granulosa cells. Fertil Steril. 2011;96(5):1246-51.e1.

14. Rosen MP, Johnstone E, McCulloch CE, et al. A characterization of the relationship of ovarian reserve markers with age. Fertil Steril. 2012;97(1):238-243.

15. de Vet A, Laven JS, de Jong FH, Themmen AP, Fauser BC. Antimüllerian hormone serum levels: a putative marker for ovarian aging. Fertil Steril. 2002;77(2):357-362.

16. Carmina E, Fruzzetti F, Lobo RA. Features of polycystic ovary syndrome (PCOS) in women with functional hypothalamic amenorrhea (FHA) may be reversible with recovery of menstrual function. Gynecol Endocrinol. 2018;34(4):301-304.

17. Rotterdam ESHRE/ASRM-Sponsored PCOS Consensus Workshop Group. Revised 2003 consensus on diagnostic criteria and long-term health risks related to polycystic ovary syndrome (PCOS). Hum Reprod. 2004;19(1):41-47.
18. Teede HJ, Misso ML, Costello MF, et al.; International PCOS Network. Recommendations from the international evidencebased guideline for the assessment and management of polycystic ovary syndrome. Fertil Steril. 2018;110(3):364-379.

19. Ferriman D, Gallwey JD. Clinical assessment of body hair growth in women. J Clin Endocrinol Metab. 1961;21:1440-1447.

20. Hatch R, Rosenfield RL, Kim MH, Tredway D. Hirsutism: implications, etiology, and management. Am J Obstet Gynecol. 1981;140(7):815-830.

21. Lie Fong S, Visser JA, Welt CK, et al. Serum anti-müllerian hormone levels in healthy females: a nomogram ranging from infancy to adulthood. J Clin Endocrinol Metab. 2012;97(12):4650-4655.

22. Long WQ, Ranchin V, Pautier P, et al. Detection of minimal levels of serum anti-Müllerian hormone during follow-up of patients with ovarian granulosa cell tumor by means of a highly sensitive enzyme-linked immunosorbent assay. J Clin Endocrinol Metab. 2000;85(2):540-544.

23. Daan NM, Louwers YV, Koster MP, et al. Cardiovascular and metabolic profiles amongst different polycystic ovary syndrome phenotypes: who is really at risk? Fertil Steril. 2014;102(5):14441451.e3.

24. Carmina E, Fruzzetti F, Lobo RA. Increased anti-Mullerian hormone levels and ovarian size in a subgroup of women with functional hypothalamic amenorrhea: further identification of the link between polycystic ovary syndrome and functional hypothalamic amenorrhea. Am J Obstet Gynecol. 2016;214(6):714. e1-714.e6.

25. Luisi S, Ciani V, Podfigurna-Stopa A, et al. Serum anti-Müllerian hormone, inhibin B, and total inhibin levels in women with hypothalamic amenorrhea and anorexia nervosa. Gynecol Endocrinol. 2012;28(1):34-38.

26. Weenen C, Laven JS, Von Bergh AR, et al. Anti-Müllerian hormone expression pattern in the human ovary: potential implications for initial and cyclic follicle recruitment. Mol Hum Reprod. 2004;10(2):77-83.

27. Pellatt L, Rice S, Mason HD. Anti-Müllerian hormone and polycystic ovary syndrome: a mountain too high? Reproduction. 2010;139(5):825-833.

28. Jonard S, Pigny P, Jacquesson L, Demerle-Roux C, Robert Y, Dewailly D. The ovarian markers of the FSH insufficiency in functional hypothalamic amenorrhoea. Hum Reprod. 2005;20(1):101-107.

29. van Elburg AA, Eijkemans MJ, Kas MJ, et al. Predictors of recovery of ovarian function during weight gain in anorexia nervosa. Fertil Steril. 2007;87(4):902-908.

30. Miller KK, Lawson EA, Mathur V, et al. Androgens in women with anorexia nervosa and normal-weight women with hypothalamic amenorrhea. J Clin Endocrinol Metab. 2007;92(4):1334-1339.

31. Bradbury RA, Lee P, Smith HC. Elevated anti-Mullerian hormone in lean women may not indicate polycystic ovarian syndrome. Aust N Z J Obstet Gynaecol. 2017;57(5):552-557. 